\title{
Altering UDP-Glucose Donor Substrate Specificity of Bacillus licheniformis Glycosyltransferase towards TDP-Glucose ${ }^{\mathrm{S}}$
}

\author{
Kye Woon Cho ${ }^{1 \dagger}$, Tae-Su Kim ${ }^{1,2 \dagger}$, Tuoi Thi Le ${ }^{1}$, Hue Thi Nguyen ${ }^{1}$, So Yeong $\mathrm{Oh}^{2}$, Ramesh Prasad Pandey ${ }^{1,2}$, \\ and Jae Kyung Sohng ${ }^{1,2 *}$ \\ ${ }^{1}$ Department of Life Science and Biochemical Engineering, Sun Moon University, Chungnam 31460, Republic of Korea \\ ${ }^{2}$ Department of Pharmaceutical Engineering and Biotechnology, Sun Moon University Chungnam 31460, Republic of Korea
}

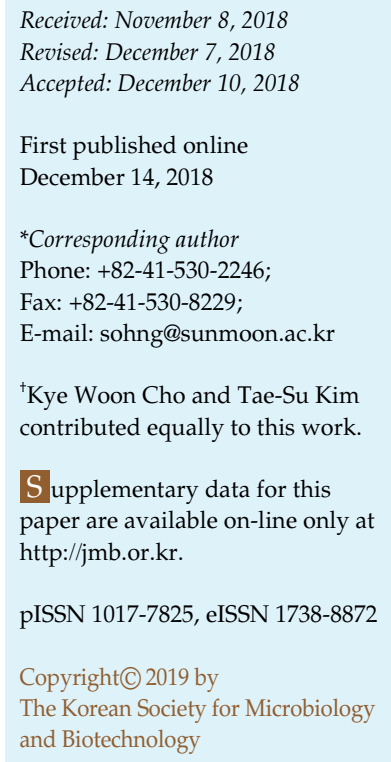

The specificity of a Bacillus licheniformis uridine diphosphate (UDP) glycosyltransferase, YjiC, was increased towards thymidine diphosphate (TDP)-sugar by site-directed mutagenesis. The Arg-282 of YjiC was identified and investigated by substituting with Trp. Conversion rate and kinetic parameters were compared between $\mathrm{YjiC}$ and its variants with several acceptor substrates such as 7-hydroxyflavone (7-HF), 4',7-dihydroxyisoflavone, 7,8-dihydroxyflavone and curcumin. Molecular docking of TDP-glucose and 7-HF with YjiC model showed pi-alkyl interaction with Arg-282 and His-14, and pi-pi interaction with $\mathrm{His}^{14}$ and thymine ring. YjiC (H14A) variant lost its glucosylation activity with TDP-glucose validating significance of His14 in binding of TDP-sugars.

Keywords: Glycosyltransferase, protein engineering, enzyme kinetics, mutation, TDP-glucose substrate specificity
Natural products (NPs), decorated with sugar residues constitute a library of compounds with a variety of biological activities. Currently used clinical compounds, such as antibiotics and anticancer agents are found to be decorated by diverse kinds of sugars. For example, anticancer (doxorubicin, calicheamicin, AT2433), antibiotics (vancomycin, erythromycin, megalomycin, kanamycin, streptomycin), antifungal (nystatin, amphotericin B, saponins), and antiparasitic (avermectins) drugs contain highly modified sugars in their structures. These sugars usually participate in the interaction between the drug and the cellular target [1]. Notably, these sugars are biosynthesized in the activated form (NDP-sugar) so that they can be used by glycosyltransferases (GTs). The phosphonucleotidyl moiety has dual purposes: serving as a recognition element for enzymes and functioning as a good leaving group during the glycosylation reaction [2]. TDPactivated sugars are the most structurally diverse class of nucleotide sugars found in nature and the preferred sugar donors in the biosynthesis of most of the bacterial glycosylated natural products [3].

Recently, a Bacillus licheniformis UDP-glycosyltransferase (UGT)-YjiC has been extensively applied for biosynthesis of various small molecule glycosides such as flavonoids [4-7], xanthone [8,9] and macrolides [10] with nonregiospecificity. In addition, YjiC showed remarkable flexibility toward five NDP-glucoses and different sugar moieties in in vitro reaction [5]. UDP-glucose is the favored substrate for YjiC. To employ YjiC for biotechnological production of microbial sugar-conjugated plant metabolites, it needs to be specific towards TDP-sugars as these are abundantly present in microbial cytosol.

In this study, we generated mutated variants of $\mathrm{YjiC}$ to explore the TDP-sugar specificity of the enzyme. By homology modeling and amino acid sequence alignment, two amino acids, His-14 and Arg-282, were identified as 
potential candidate amino acids for TDP-sugar specificities. We generated H14A and R282W mutated variants of YjiC and studied their role to get insight on TDP-glucose binding and glucosylation activity. The YjiC and its variants were expressed to soluble fraction, purified by His-tag affinity resin and quantified using Bradford assay [11-14].

Prior to enzymatic assay, the in silico analysis of YjiC was performed by docking 7-hydroxyflavone (7-HF) and TDP-glucose and comparing the amino acid sequence with previously known UGTs. The BLAST search in Discovery Studio 2018 was used for searching homologous structure of YjiC based on its amino acid sequence. Two structures, OleD (UGT) (PBD number: 2IYF) and CalG4 (TDPglycosyltranserase; TGT) (PDB code: 3IA7) with the highest identity $(40 \%)$ were chosen as the templates for building homology models of YjiC using Discovery Studio 2018 (DS 2018; BIOVIA, USA). The coordinates for UDP were obtained from 2IYF and positioned as in the template. The predicted YjiC model displayed the GT-B fold typical of several GT families including GT-1, consisting of two Rossmann-like $\beta / \alpha / \beta$ domains that are not tightly associated. Catalytic diad His-14 and Asp-110 of YjiC were well conserved based on comparison of the modeled YjiC structure and structure of OleD. The crystal structure of several UGTs showed highly conserved residues of Trp and Val (Ala) for uridine moiety base recognition of UDPsugars. In the crystal structure of OleD bound UDP, the uridine stacks against the aromatic side chain of Trp-289 and forms hydrogen bonds with Val-290. Similar interactions are observed in the other GT-1 enzymes that are not highly
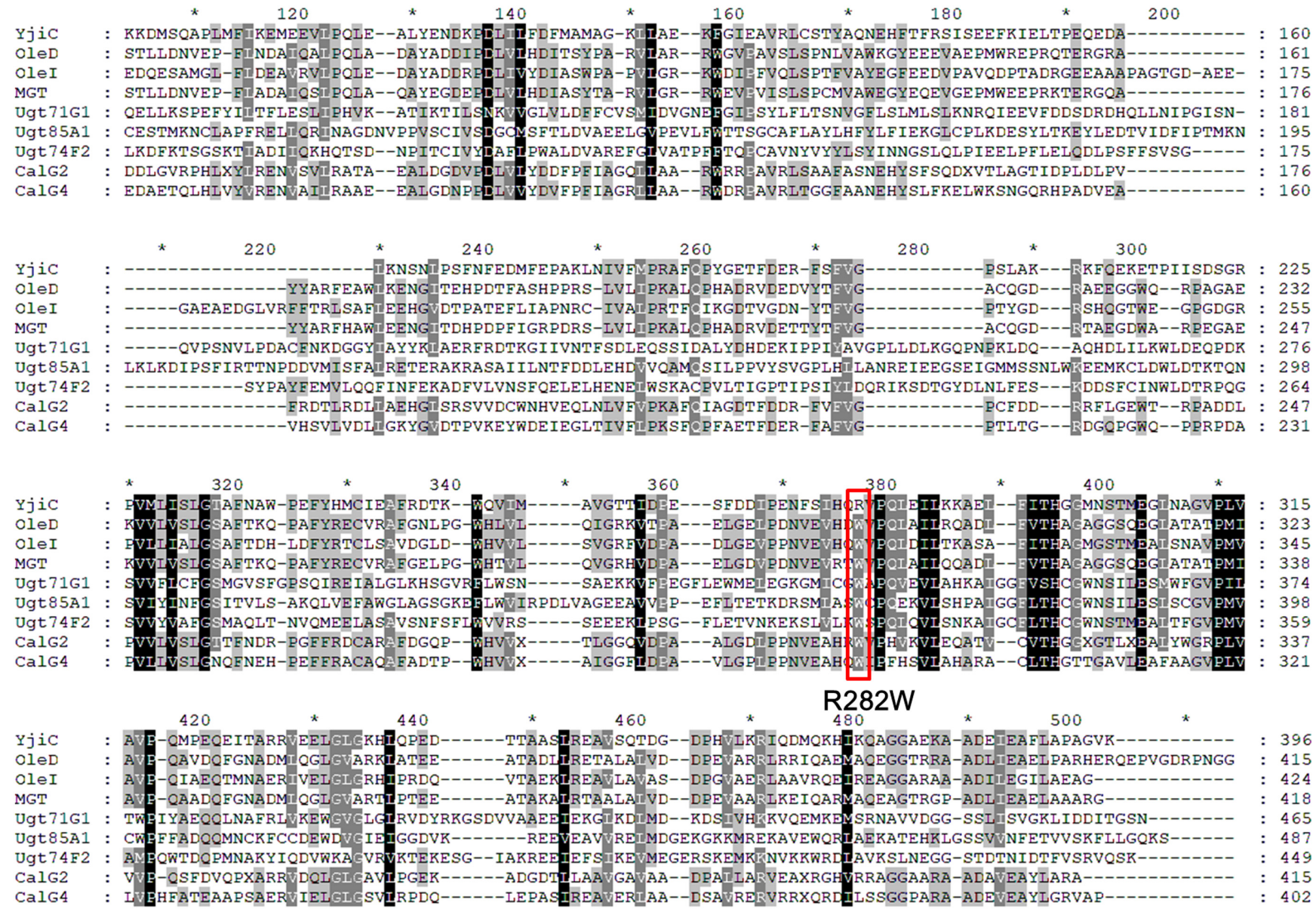

Fig. 1. Amino acid sequence alignment of various glycosyltransferase from Bacillus licheniformis, Arabidopsis thaliana, Micromonospora echinospora, Medicago truncatula, Strptomyces lividans, Streptomyces antibioticus to check conserved region of W282. The alignment was performed using Clustal X. The aligned amino acid sequences are YjiC (WP_003182014.1) from Bacillus licheniformis, UGT71G1 (2ACW_B), UGT74F2 (OAP07463.1), and UGT85A1 (OAP13723.1) from Arabidopsis thaliana, The OleI (AAC12648.1), OleD (ABA42119.2) from Streptomyces antibioticus, MGT (ABA28305.2) from Strptomyces lividans and CalG2(3RSC_A), CalG4 (3IA7_A) from Micromonospora echinospora. Conserved W282 residues are marked by a black diamond below the alignment. 
related to OleD, for example, in UGT71G1, the equivalent residues to OleD are Trp-289 (Trp-339) and Val-290 (Ala340), whereas in VvGT1, the corresponding amino acids are Trp-332 and Ala-333 [15-17]. Similar to the UGTs, some TGTs such as CalG2 and CalG4, have conserved Trp-Val, Trp-Ile, respectively, in the same position. These amino acids could play the same function like UGTs (pi-pi interaction). Interestingly, in $\mathrm{YjiC}$ model, two equivalent residues to these amino acids were Arg-282 basic amino acid and Val283 (Fig. 1). Therefore, the Arg-282 of YjiC was selected for substitution by the aromatic amino acid Trp.

The donor and acceptor binding pattern can influence the catalytic activity of GT [18-22]. Firstly, UDP-glucose binds to the donor binding site of GT in catalytic manner followed by acceptor substrate binding to the active site in proper orientation. In $\mathrm{YjiC}$, the interactions with TDPglucose include $p i$-alkyl interaction between Arg-282 with thymine ring, and His-14 with methyl group of thymine ring (Fig. 2A). In addition His-14 forms pi-pi interactions with thymine ring of TDP-glucose (Fig. 2A). Furthermore, the amino acids Thr-234, Thr-263, Gln-281, Arg-282, Met-
301 and Asn-302 showed hydrogen bonding network with TDP-glucose (Fig. 2A). Also, R282W substitution led to breaking of pi-alkyl interactions and the hydrogen bonds with thymine ring (Figs. 2A and 2B). However, the same pialkyl and pi-pi interactions are shown in His-14 (Figs. 2A and 2B). Thus, His-14 and Arg-282 residues are very important for proper binding of TDP-glucose in YjiC. $\mathrm{R} 282 \mathrm{~W}$ changed the binding orientation of TDP-glucose due to breaking the pi-alkyl interaction and hydrogen bonds (Fig. 2C). As a result, R282W distance was decreased from $3.39 \AA$ to $2.97 \AA$ in between donor $\alpha$ carbon of TDPglucose and oxygen of 7-hydroxyl group of 7-HF (Figs. 2B and 2D). The distance between the nitrogen of His-16 and the proton of 7-hydroxyl group of 7-HF was decreased from $3.36 \AA$ to $2.52 \AA$ (Figs. 2B and 2D). Direct displacement $\mathrm{SN}_{2}-$ like mechanism is important for inverting GTs. So, reduced distance influences the conversion rate of 7-HF while using TDP-glucose donor in the reaction, while $\mathrm{R} 282 \mathrm{~W}$ is anticipated to exhibit more favorable glycosylation reaction using TDP-glucose than $\mathrm{YjiC}$. Moreover, pi-alkyl interactions can be seen between Ala-13 with A ring of 7-HF, Ala-13,
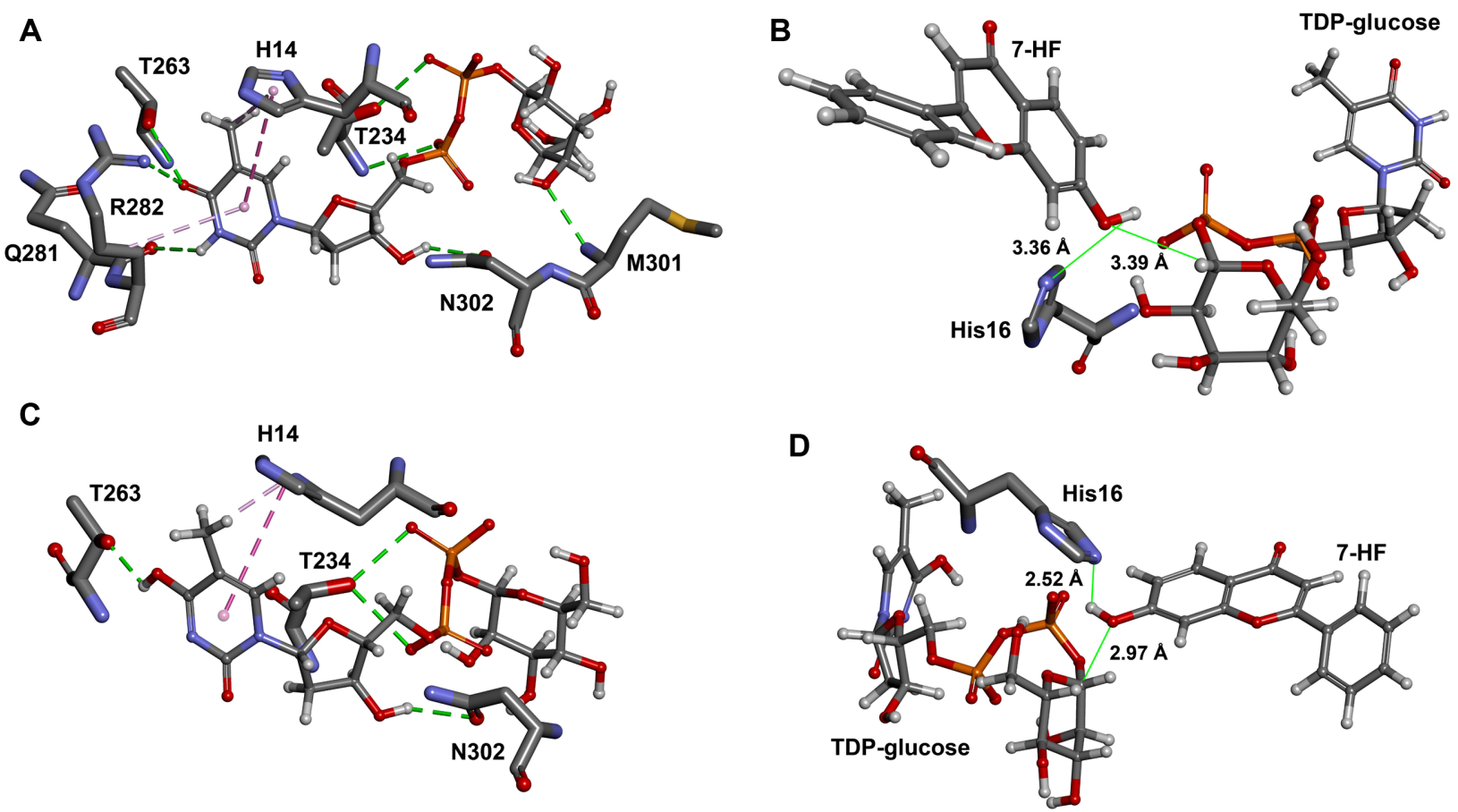

Fig. 2. Molecular docking of TDP-glucose and 7-HF with YjiC model.

(A) TDP-glucose docking in the NDP-sugar binding site of YjiC. (B) TDP-glucose and 7-HF docking in the donor and acceptor substrates binding pocket of YjiC. (C) TDP-glucose docking in the NDP-sugar binding site of R282W. (D) TDP-glucose and 7-HF docking in the substrates binding pocket of R282W. All atoms are colored to standard coloring. Amino acid residues are represented by the stick model, whereas bound donor and acceptor substrate atoms are shown by the ball-and-stick model. Bond lengths are displayed in $\AA$. Hydrogen atoms were removed in amino acid residues to improve clarity, and images were made by Discovery Studio 2018. 

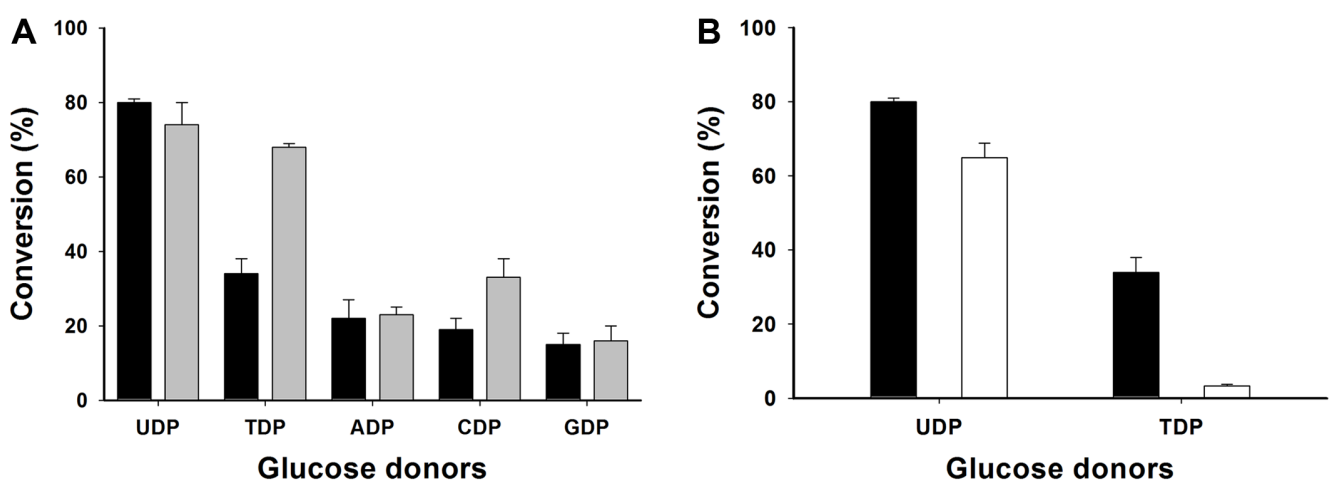

Fig. 3. (A) The conversion of 7-HF to glucoside by R282W (in gray) with various NDP-glucoses. (B) The conversion of 7-HF to glucoside by H14A (in white) and wt-YjiC (in black) with U(T)DP-glucose.

Leu60, Met-71, Ala-80, Pro-81, Ile-145 with B ring and Leu60 with $\mathrm{C}$ ring, respectively (Fig. S1). Identical interactions were also observed in R282W mutant (Fig. S1). These results clearly indicate that only TDP-glucose binding will be influenced but not the binding of 7-HF in R282W variant in comparison to $\mathrm{YjiC}$.

H14A and R282W were generated, cloned, and expressed in Escherichia coli BL21 (DE3) along with pET302/NT-His vector. Analysis by SDS/PAGE demonstrated that isolated $\mathrm{H} 14 \mathrm{~A}$ and $\mathrm{R} 282 \mathrm{~W}$ both exhibited protein size in the range of $46 \mathrm{kDa}$, the same molecular weight as wild-type YjiC (Fig. S2). Purified YjiC and R282W were assayed with 7-HF as acceptor and various NDP-glucoses (U/T/C/A/GDPglucose) as donors as described in supporting methods. In case of wild type, UDP-glucose resulted in the highest conversion (79.9\%) of 7-HF to its glucoside formation among other NDP-sugars (Fig. 3A). The conversion of 7-HF to its glucoside was 33.6, 19.4, 22.1 and 15\% when TDP, CDP, $\mathrm{ADP}$ and GDP-glucose were used in the reaction mixture, respectively. Only $5.5 \%, 0.4 \%, 13.8 \%$, and $1 \%$ conversion rate was increased in case of UDP-glucose, CDP-glucose, ADP-glucose and CDP-glucose while comparing between wild type and R282W (Fig. 3A and Table S1A). But in case of TDP-glucose, there was $34.4 \%$ increase in the conversion rate (Fig. 3A and Table S1A). Thus, we were interested in identification of the governing factor that can influence on the increased conversion rate of R282W using TDP-glucose. We also performed kinetic studies of R282W mutant. Reaction condition was optimized for enzyme kinetic studies. The highest conversion of 7-HF to glycosylated products was observed at $\mathrm{pH} 9$ (Fig. S3A) and optimal $\mathrm{MgCl}_{2}$ concentration of $10 \mathrm{mM}$ (Fig. S3B). These results suggested that the highest conversion of 7-HF is relatively better under basic conditions of reaction buffer $(\mathrm{pH} 9-10)$ than in acidic conditions $(\mathrm{pH}$ 6-7) (Fig. S3A). YjiC and R282W exhibited $K_{\mathrm{m}}$ and $V_{\max }$ values of $2.07 \mathrm{mM}, 2.19 \mathrm{mM}$, and $23.02 \mu \mathrm{mol} / \mathrm{min} / \mu \mathrm{g}$ protein, $85.61 \mu \mathrm{mol} / \mathrm{min} / \mu \mathrm{g}$ protein toward TDP-glucose, respectively (Figs. S4A and S4B). It clearly shows that TDPglucose conversion was enhanced due to increase in catalytic efficiency in R282W but not binding affinity of donor substrate. It is coherent to the result with in silico docking analysis. H14A was created for better understanding of interaction between TDP-glucose and YjiC. Enzyme reaction was performed with UDP-glucose and TDP-glucose using $\mathrm{H} 14 \mathrm{~A}$. In case of UDP-glucose, the activity was $81 \%$ when compared with YjiC (Fig. 3B or Table S1B). In contrast, only $9 \%$ activity was observed in case of TDP-glucose (Fig. 3B or Table S1B). Our results demonstrated that His-14 has more effect on the conversion of TDP-glucose in YjiC. In the H14A mutant, there was loss of two pi-pi and pi-alkyl interactions with thymine ring (Fig. 2A). So, TDP-glucose was not properly bound to the active site resulting in a loss of enzyme activity.

Enzyme reactions were also performed with various other acceptor molecules (4',7-dihydroxy isoflavone, 7.8dihydroxyflavone, curcumin) to confirm the reaction pattern of R282W (Fig. 4 and Tables S1C-S1E). Enzyme reaction was performed based on previous reports [23-25]. In case of UDP-glucose, the conversion rate of all glucosylated products was similar $(1 \sim 4 \%$ different $)$ compared to YjiC and R282W (Fig. 4 and Tables S1C-S1E). In contrast there was enhanced conversion rate (4 39\%) with TDP-glucose (Fig. 4 and Tables S1C-S1E). While TDPglucose was used for enzyme reaction, R282W mutant and YjiC exhibited $47 \%, 28 \%$, and $46 \%$ increment on total conversion of 4 , 7 -dihydroxy isoflavone, 7.8-dihydroxyflavone 
A

4',7-dihydroxyis oflavone

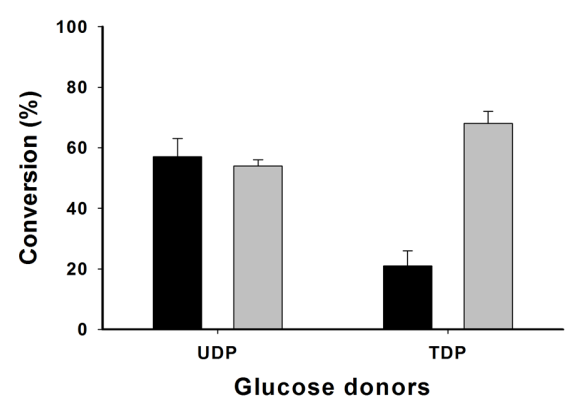

B

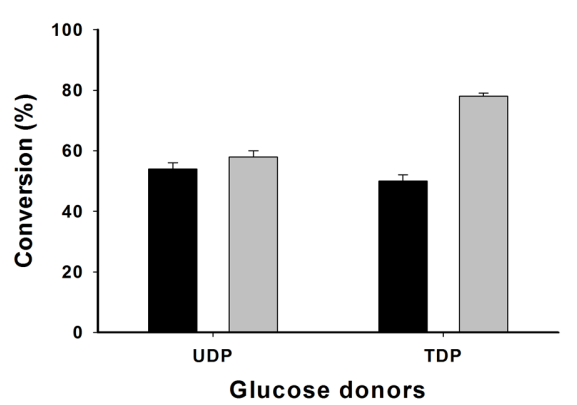

Curcumin

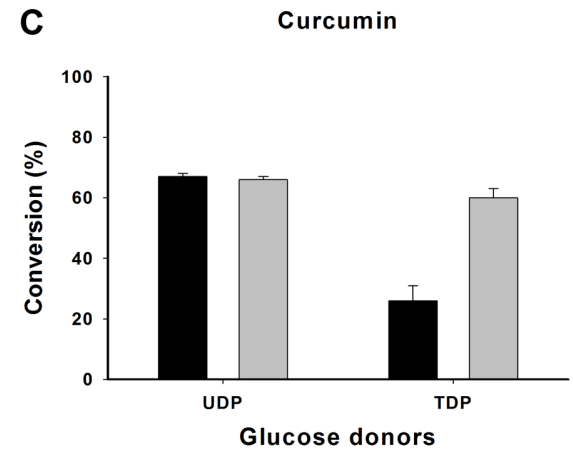

Fig. 4. The comparison of glycosylation reactions by YjiC-R282W with U(T)DP-glucose.

(A) 4',7-Dihydroxy-isoflavone, (B) 7,8-Dihydroxyflavone, and (C) Curcumin.

and curcumin, respectively. Again, this result clearly demonstrates the increased affinity of R282W mutant with TDP-glucose.

We further characterized the glucosylated reaction products of 7-HF, 4',7-dihydroxy isoflavone, 7,8-dihydroxyflavone and curcumin with U(T)DP-glucose by HPLC-PDA (Fig. S5 and Table S2) and QTOF-HR ESI/MS (Figs. S6-S9 and Table S3). The HR ESI/MS analysis confirmed the product to be 7-HF 7-O- $\beta$-D-glucoside (H1, $[\mathrm{M}+\mathrm{H}]^{+} \mathrm{m} / \mathrm{z}^{+} \sim 401.1231$ ) as 7-HF contains a single hydroxyl group at the C-7 position. The mass analysis identified a $4^{\prime}, 7$-dihydroxy isoflavone (I1) $\left(t_{R}=13.1 \mathrm{~min},[\mathrm{M}+\mathrm{H}]^{+} \mathrm{m} / \mathrm{z}^{+} \sim 255.0640\right)$, 4$O$-glucoside derivative of $4^{\prime}, 7$-dihydroxy isoflavone (I2, $t_{R}$ $\left.=10.6 \mathrm{~min},[\mathrm{M}+\mathrm{H}]^{+} \mathrm{m} / \mathrm{z}^{+} \sim 417.1179\right)$, and 7-O-glucoside derivative of 4',7-dihydroxy isoflavone ( $\mathrm{I} 3, t_{R}=10.2 \mathrm{~min}$, $\left.[\mathrm{M}+\mathrm{H}]^{+} \mathrm{m} / \mathrm{z}^{+} \sim 417.1169\right)$. Similarly, with 7,8-dihydroxyflavone, a 7-O-glucoside derivative ( $\mathrm{F} 1, t_{R}=11.5 \mathrm{~min},[\mathrm{M}+\mathrm{H}]^{+} \mathrm{m} / \mathrm{z}^{+}$ 417.1181), 8-O-glucoside derivative $\left(\mathrm{F} 2, t_{R}=11.2 \mathrm{~min}\right.$, $\left.[\mathrm{M}+\mathrm{H}]^{+} \mathrm{m} / \mathrm{z}^{+} \sim 417.1172\right)$ were characterized based on previous reports [24]. With curcumin, a curcumin $4^{\prime}-O-\beta$ glucoside $\left(\mathrm{C} 1, t_{R}=13.4 \mathrm{~min},[\mathrm{M}+\mathrm{H}]^{+} \mathrm{m} / \mathrm{z}^{+} \sim 531.1848\right)$, a curcumin $4^{\prime}, 4^{\prime \prime}$ di-O- $\beta$-glucoside $\left(\mathrm{C} 2, t_{R}=11.1 \mathrm{~min}\right.$, $[\mathrm{M}+$ $\mathrm{H}]^{+} \mathrm{m} / \mathrm{z}^{+} \sim 693.2390$ ) were detected in the reaction mixture.

In conclusion, we tuned donor substrate specificity of YjiC from preferred UDP-glucose to TDP-glucose by sitedirected mutagenesis. This study provides evidence for better understanding of $\mathrm{YjiC}$ catalytic activity and donor substrate specificity. Two residues (Arg 282 and His14) identified by in silico study and characterized by mutagenesis studies present evidence for promiscuous activity of YjiC with diverse donor substrates. The study also provides information for further engineering GT by saturated mutagenesis for target NDP-sugar specificity in the near future.

\section{Acknowledgments}

This work was supported by a grant from the NextGeneration BioGreen 21 Program (SSAC, grant\#: PJ013137), Rural Development Administration, Republic of Korea. In addition, this research was supported by the Basic Science Research Program through the National Research Foundation of Korea (NRF) funded by the Ministry of Education (2017R1A6A3A11035875)

\section{Conflict of Interest}

The authors have no financial conflicts of interest to declare.

\section{Reference}

1. Thibodeaux CJ, Melancon CE 3rd, Liu HW. 2008. Naturalproduct sugar biosynthesis and enzymatic glycodiversification. Angew. Chem. Int. Ed. Engl. 47: 9814-9859.

2. Salas JA, Mendez C. 2007. Engineering the glycosylation of natural products in actinomycetes. Trends Microbiol. 15: 219232.

3. Thibodeaux CJ, Melancon CE 3rd, Liu HW. 2008. Naturalproduct sugar biosynthesis and enzymatic glycodiversification. Angew. Chem. Int. Ed. Engl. 47: 9814-9859.

4. Pandey RP, Li TF, Kim EH, Yamaguchi T, Park YI, Kim JS, et al. 2013. Enzymatic synthesis of novel phloretin glucosides. Appl. Environ. Microbiol. 79: 3516-3521.

5. Pandey RP, Parajuli P, Koirala N, Park JW, Sohng JK. 2013. Probing 3-hydroxyflavone for in vitro glycorandomization of flavonols by YjiC. Appl. Environ. Microbiol. 79: 6833-6838.

6. Pandey RP, Gurung RB, Parajuli P, Koirala N, Tuoi le T, Sohng JK. 2014. Assessing acceptor substrate promiscuity of YjiC-mediated glycosylation toward flavonoids. Carbohydr. Res. 393: 26-31. 
7. Gurung RB, Kim EH, Oh TJ, Sohng JK. 2013. Enzymatic synthesis of apigenin glucosides by glucosyltransferase (YjiC) from Bacillus licheniformis DSM 13. Mol. Cells 36: 355-361.

8. Le TT, Pandey RP, Gurung RB, Dhakal D, Sohng JK. 2014. Efficient enzymatic systems for synthesis of novel alphamangostin glycosides exhibiting antibacterial activity against Gram-positive bacteria. Appl. Microbiol. Biotechnol. 98: 85278538.

9. Kim TS, Le TT, Nguyen HT, Cho KW, Sohng JK. 2018. Mutational analyses for product specificity of $\mathrm{YjiC}$ towards alpha-mangostin mono-glucoside. Enzyme Microb. Technol. 118: $76-82$.

10. Wu CZ, Jang JH, Woo M, Ahn JS, Kim JS, Hong YS. 2012. Enzymatic glycosylation of nonbenzoquinone geldanamycin analogs via Bacillus UDP-glycosyltransferase. Appl. Environ. Microbiol. 78: 7680-7686.

11. Cheng Y, Wei H, Sun R, Tian Z, Zheng X. 2016. Rapid method for protein quantitation by Bradford assay after elimination of the interference of polysorbate 80. Anal. Biochem. 494: 37-39.

12. Kruger NJ. 1994. The Bradford method for protein quantitation. Methods Mol. Biol. 32: 9-15.

13. Hammond JB, Kruger NJ. 1988. The bradford method for protein quantitation. Methods Mol. Biol. 3: 25-32.

14. Kim TS, Patel SK, Selvaraj C, Jung WS, Pan CH, Kang YC, et al. 2016. A highly efficient sorbitol dehydrogenase from Gluconobacter oxydans $G 624$ and improvement of its stability through immobilization. Sci. Rep. 6: 33438.

15. Bolam DN, Roberts S, Proctor MR, Turkenburg JP, Dodson EJ, Martinez-Fleites C, et al. 2007. The crystal structure of two macrolide glycosyltransferases provides a blueprint for host cell antibiotic immunity. Proc. Natl. Acad. Sci. USA 104: 5336-5341.

16. Shao H, He X, Achnine L, Blount JW, Dixon RA, Wang X. 2005. Crystal structures of a multifunctional triterpene/ flavonoid glycosyltransferase from Medicago truncatula. Plant Cell 17: 3141-3154.

17. Offen W M-FC, Yang M, Kiat-Lim E, Davis BG, Tarling CA, Ford CM, et al. 2006. Structure of a flavonoid glucosyltransferase reveals the basis for plant natural product modification. EMBO J. 25: 1396-1405.

18. He XZ, Wang X, Dixon RA. 2006. Mutational analysis of the Medicago glycosyltransferase UGT71G1 reveals residues that control regioselectivity for (iso)flavonoid glycosylation. J. Biol. Chem. 281: 34441-34447.

19. Osmani SA, Bak S, Moller BL. 2009. Substrate specificity of plant UDP-dependent glycosyltransferases predicted from crystal structures and homology modeling. Phytochemistry 70: 325-347.

20. Kubo A, Arai Y, Nagashima S, Yoshikawa T. 2004. Alteration of sugar donor specificities of plant glycosyltransferases by a single point mutation. Arch. Biochem. Biophys. 429: 198-203.

21. Hoffmeister D, Wilkinson B, Foster G, Sidebottom PJ, Ichinose K, Bechthold A. 2002. Engineered urdamycin glycosyltransferases are broadened and altered in substrate specificity. Chem. Biol. 9: 287-295.

22. Gantt RW, Peltier-Pain P, Singh S, Zhou M, Thorson JS. 2013. Broadening the scope of glycosyltransferase-catalyzed sugar nucleotide synthesis. Proc. Natl. Acad. Sci. USA 110: 7648-7653.

23. Pandey RP, Parajuli P, Koirala N, Lee JH, Park YI, Sohng JK. 2014. Glucosylation of isoflavonoids in engineered Escherichia coli. Mol. Cells 37: 172-177.

24. Pandey RP, Parajuli P, Pokhrel AR, Sohng JK. 2018. Biosynthesis of novel 7,8-dihydroxyflavone glycoside derivatives and in silico study of their effects on BACE1 inhibition. Biotechnol. Appl. Biochem. 65: 128-137.

25. Gurung RB, Gong SY, Dhakal D, Le TT, Jung NR, Jung HJ, et al. 2017. Synthesis of curcumin glycosides with enhanced anticancer properties using one-pot multienzyme glycosylation technique. J. Microbiol. Biotechnol. 27: 1639-1648. 